\title{
Ultrafast Probe of Carrier Diffusion and Nongeminate Processes in a Single CdSSe Nanowire
}

\author{
Peter S. Eldridge, ${ }^{1}$ Jolie C. Blake, ${ }^{1}$ and Lars Gundlach ${ }^{1,2}$ \\ ${ }^{1}$ Department of Chemistry and Biochemistry, University of Delaware, Newark, DE 19716, USA \\ ${ }^{2}$ Department of Physics and Astronomy, University of Delaware, Newark, DE 19716, USA \\ Correspondence should be addressed to Lars Gundlach; larsg@udel.edu
}

Received 31 October 2014; Accepted 19 February 2015

Academic Editor: Pedro D. Vaz

Copyright (c) 2015 Peter S. Eldridge et al. This is an open access article distributed under the Creative Commons Attribution License, which permits unrestricted use, distribution, and reproduction in any medium, provided the original work is properly cited.

\begin{abstract}
We measure ultrafast carrier dynamics in a single CdSSe nanowire at different excitation fluences using an ultrafast Kerr-gated microscope. The time-resolved emission exhibits a dependence on excitation fluence, with the onset of the emission varying on the picosecond time scale with increasing laser power. By fitting the emission to a model for amplified spontaneous emission (ASE), we are able to extract the nonradiative carrier recombination lifetime and nongeminate recombination constant. The extracted nongeminate recombination constant suggests that our measurement technique allows the access to the nondiffusion limited recombination regime in nanowires with low carrier mobility.
\end{abstract}

\section{Introduction}

With the vast progress in chemical synthesis of semiconductor nanostructures, there is a need to understand carrier transport in the plethora of available morphologies. Ternary alloy semiconductors, such as $\mathrm{CdS}_{x} \mathrm{Se}_{1-x}$, are particularly intriguing for both scientific studies and technological applications. As the bandgap of $\mathrm{CdS}_{x} \mathrm{Se}_{1-x}$ can span the entire visible spectrum through alloy variation, it has potential applications as a light harvester in solar cells and a tunable wavelength source in semiconductor nanolasers [1-3]. The high defect density inherent to ternary alloys in comparison to their binary counterparts has led to reports of increased density of nonradiative recombination centres (ShockleyRead-Hall states) in AlGaAs [4] while exciton localisation in CdSSe nanobelts has been attributed to bandgap variation along the nanobelt [5]. As compositional variations can occur on the single particle level for CdSSe, compositional and morphological variations within an ensemble of particles complicate interpretation of ensemble averaged measurements, making the need for single particle measurements all the more pertinent for carrier dynamics studies.
Here, we investigate early luminescence dynamics in a single CdSSe nanowire at different excitation intensities using a Kerr-gated ultrafast microscope [6]. The shortlived, nonlinear emission is assigned to amplified spontaneous emission (ASE). Fitting the emission to a nonlinear model allows us to extract information on the impurity mediated nonradiative carrier lifetime and the nongeminate recombination coefficient. The nongeminate recombination coefficient demonstrates that our measurement technique allows the access to carrier dynamics on short enough timescales and high enough densities that recombination is not diffusion-controlled. This therefore shows that the majority of the excited carriers recombine before significant impurity, phonon, or defect scattering.

\section{Materials and Methods}

CdSSe nanowires were grown by the vapour liquid solid (VLS) method detailed in the previously published work $[7,8]$. This technique is known to produce single crystalline nanowires with wurtzite phase and ensemble XRD measurements (not shown) are in agreement with the nanowires 
having wurtzite crystal structure. Nanowires were drop-cast onto transmission electron microscope (TEM) grids to allow different characterisation techniques to be conducted on the same nanowire. Initial characterization of the nanowires involved fluorescence microscopy with an Olympus IX70 and SEM using a Hitachi S4700.

Figure 1(a) shows a true colour micrograph of the chosen nanowire for the study. A faint outline of the TEM grid is visible on the right-hand side of the micrograph due to scattered light. Using the spectral properties of the colour filters in the charged coupled device (CCD) camera of the IX70, we can map a colour from the bitmap image to a particular emission wavelength (Figure 1(b)) [8]. The emission wavelengths can then be used to gauge composition variations along the length of the wire. Assuming that band edge emission dominates [5], the emission wavelength of $\sim 563 \mathrm{~nm}$ suggests a composition of $\mathrm{CdS}_{0.8} \mathrm{Se}_{0.2}$, using the compositional dependence found in [9], with no more than 5\% variation along the length of the wire. Figures 1(c) and 1(d) are SEM images of the nanowire. The images reveal that the surface is smooth, with no debris or significant morphology changes. From these images, we can deduce that the nanowire studied is around $200 \mathrm{~nm}$ thick and $500 \mathrm{~nm}$ wide with a length of $200 \mu \mathrm{m}$. Figure 1(e) shows the EDS spectra from the sample ensemble. From these spectra, the calculated ensemble average composition is $\mathrm{CdS}_{0.64} \mathrm{Se}_{0.36}$. This is in agreement with the colour space analysis (Figure 1(b)), considering particle to particle composition variation and experimental uncertainty.

Both time-resolved and time-integrated luminescence measurements were taken using the Kerr-gated microscope [6]. In brief, the microscope is similar to a standard fluorescence microscope, but between the microscope objective (36x magnification, $\mathrm{NA}=0.52$ ) and the detector (CCD camera), two additional objectives and an optical Kerr-gate are placed. The two additional objectives form a conjugate image plane at the position of the Kerr medium within the Kerr-gate. The gate comprises crossed linear polarisers surrounding the Kerr medium, a $0.5 \mathrm{~mm}$ thick piece of Yttrium aluminium garnet (YAG). Without the presence of a gate pulse, the crossed polarisers prevent any light from reaching the detector. Emission can be detected either through the rotation of the second polariser (open gate) to measure steady state fluorescence or through a gate pulse that changes the polarisation state of the luminescence via the Kerr-effect in the YAG crystal (closed gate). Therefore, by changing the time delay between the pump and gating pulses, the emission can be time-resolved as the polarisation of the light is only changed for luminescence which overlaps in time with the gate pulse.

The laser system used to power the microscope consists of an output from a commercial Ti:sapphire oscillator (Coherent Mantis) which is amplified in a commercial $10 \mathrm{kHz}$ amplifier (Coherent Legend Elite) producing $38 \mathrm{fs}$ pulses at $800 \mathrm{~nm}$. Half is used as the gating beam and is focused onto the YAG crystal, with a spot diameter of $\sim 200 \mu \mathrm{m}$. The other half pumps a homebuilt NOPA which generates $30 \mathrm{fs}$ pulses at $580 \mathrm{~nm}$ which are then frequency-doubled to excite the sample at $290 \mathrm{~nm}$, with a beam diameter of $80 \mu \mathrm{m}$ at the sample position. The all-reflective objectives allow a temporal resolution below $100 \mathrm{fs}$ and a submicron spatial resolution.

\section{Results and Discussion}

Figure 2(a) shows an open gate false-colour luminescence image of the nanowire from Figure 1 under an excitation fluence of $3.5 \mathrm{~mJ} / \mathrm{cm}^{2}$. At several positions along the nanowire, there are regions of high emission, as seen by the yellow colour on the image. Figure 2(b) shows the integrated intensity from two such positions along the nanowire (the red and green squares in Figure 2(a)) on a log-log plot as the fluence is increased from 2 to $6.5 \mathrm{~mJ} / \mathrm{cm}^{2}$. At first, there is a nonlinear increase with power, which flattens off above $4.4 \mathrm{~mJ} / \mathrm{cm}^{2}$, marked by the dotted line. By fitting the nonlinear increase to a power law-fit, we see a near cubic growth in the luminescence intensity with power, which is higher than that expected for either free electron and hole or exciton mediated recombination [10]. Using the data from [11], we estimate an absorption coefficient for $\mathrm{CdS}_{0.8} \mathrm{Se}_{0.2}$ at $290 \mathrm{~nm}$ of $2 \times 10^{4} \mathrm{~cm}^{-1}$ and carrier densities of 5-10 $\times$ $10^{19} \mathrm{~cm}^{-3}$. As these densities are above the Mott transition for both CdSe and CdS, at these excitation fluences, the carriers are predominantly free electron and holes. As the power is increased further, there is a reduction in the nonlinearity, as seen by the change in the slope of the integrated intensity at powers beyond the dotted line in Figure 2(b). This could be due to either carrier heating or Auger recombination. For the rest of the paper, we limit our attention to the ASE regime (fluences $<4.5 \mathrm{~mJ} / \mathrm{cm}^{2}$ ) and resolve the emission in time.

The Kerr-gate is closed and time-resolved images at different pump-probe delays are recorded. By integrating the emission at the location of the green box in Figure 2(a) for each image, we obtain the emission intensity as a function of time. Figure 3(a) is a plot of the recorded transients for two different excitation fluences, $2.2 \mathrm{~mJ} / \mathrm{cm}^{2}$ (closed grey markers) and $4.4 \mathrm{~mJ} / \mathrm{cm}^{2}$ (open blue markers). The low fluence emission is broad in time and peaks at around $3.5 \mathrm{ps}$. At the higher fluence, the emission intensity has increased and forms a sharp peak at early times ( 2 ps) with a second peak at later times. The onset of the emission shifts to earlier times with increasing power. This is seen clearly in Figure 3(b), where the dashed grey line plots the onset of the emission as a function of laser power. The false-colour image in Figure 3(b) and the solid lines in Figure 3(a) are the results from the fitting of the data with the ASE model described below.

The rate model for ASE, discussed in detail in the previous work [12], is described by the following first-order nonlinear coupled differential equations:

$$
\begin{aligned}
\frac{d}{d t} N_{1}(t)= & A V_{p}(t)-\gamma_{r} N_{1}(t) \\
\frac{d}{d t} N_{2}(t)= & \gamma_{r} N_{1}(t)-B N_{2}(t) \Phi(t)-\gamma_{s} N_{2}(t) \\
& -\gamma_{g} N_{2}(t)^{2}-\gamma_{a} N_{2}(t)^{3} \\
\frac{d}{d t} \Phi(t)= & B N_{2}(t) \Phi(t)-\gamma_{\Phi} \Phi(t),
\end{aligned}
$$




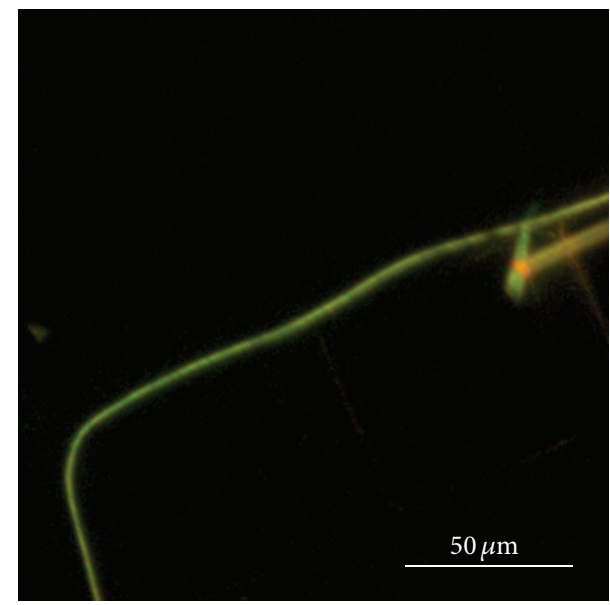

(a)

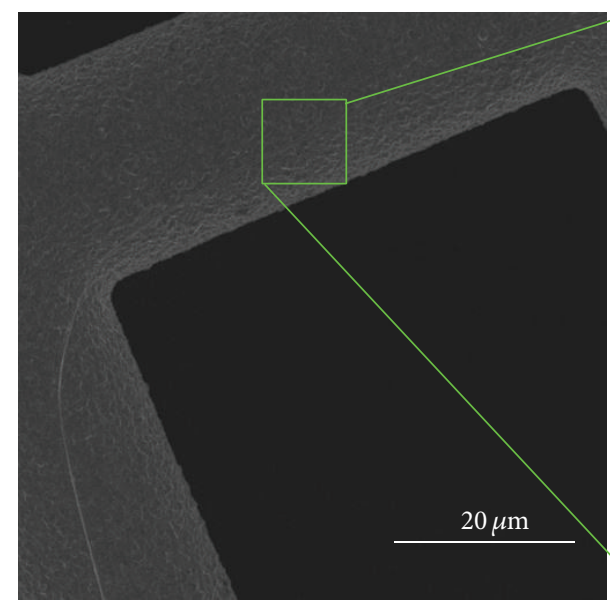

(c)

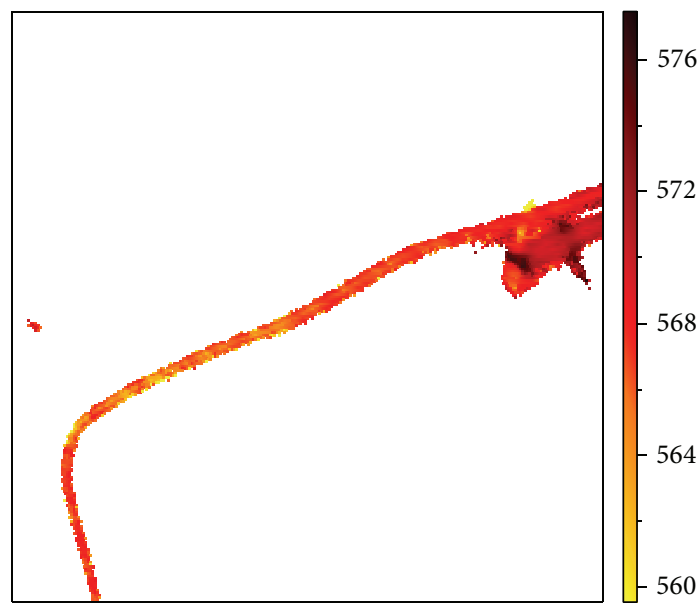

(b)

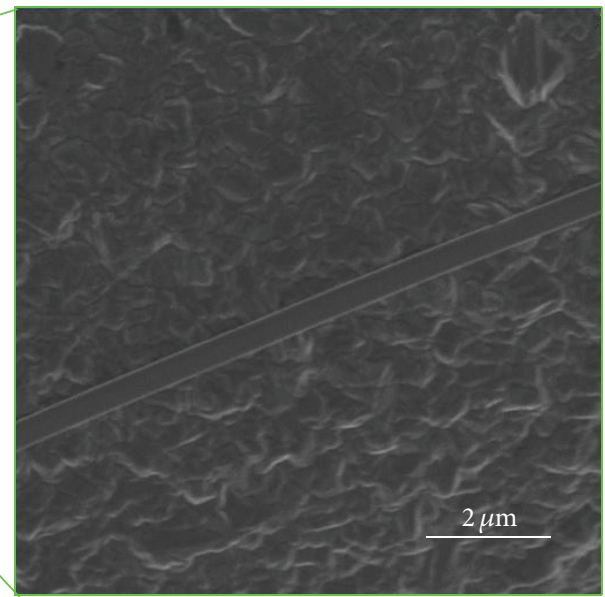

(d)

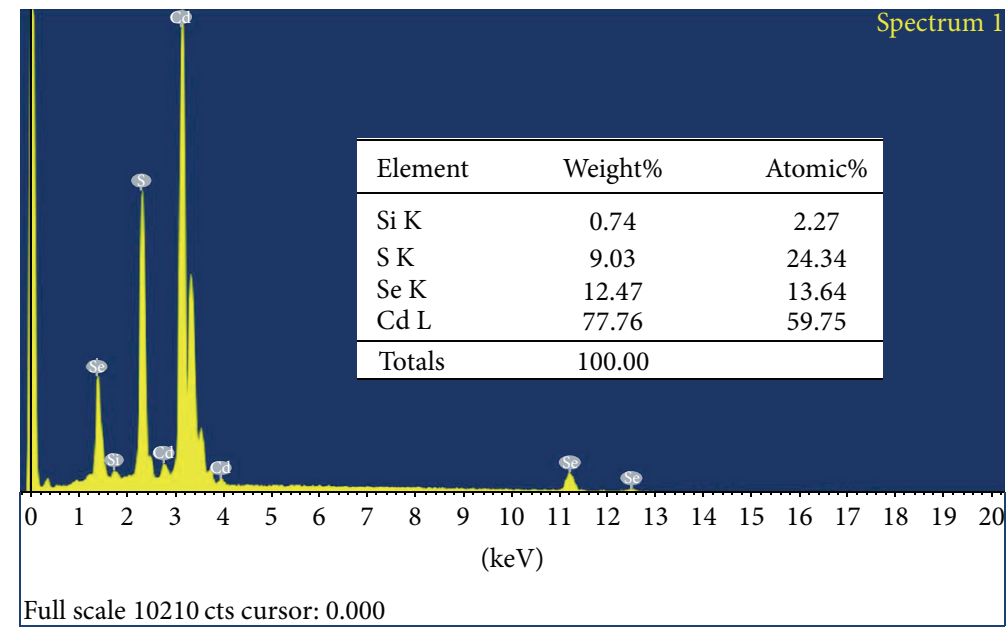

(e)

Figure 1: (a) A fluorescence micrograph of the studied CdSSe nanowire on a TEM grid. (b) The results of a colour space analysis of the micrograph displaying the extracted emission wavelength in nanometers. ((c) and (d)) SEM images of the same nanowire. (e) EDS measurement for the sample ensemble. 


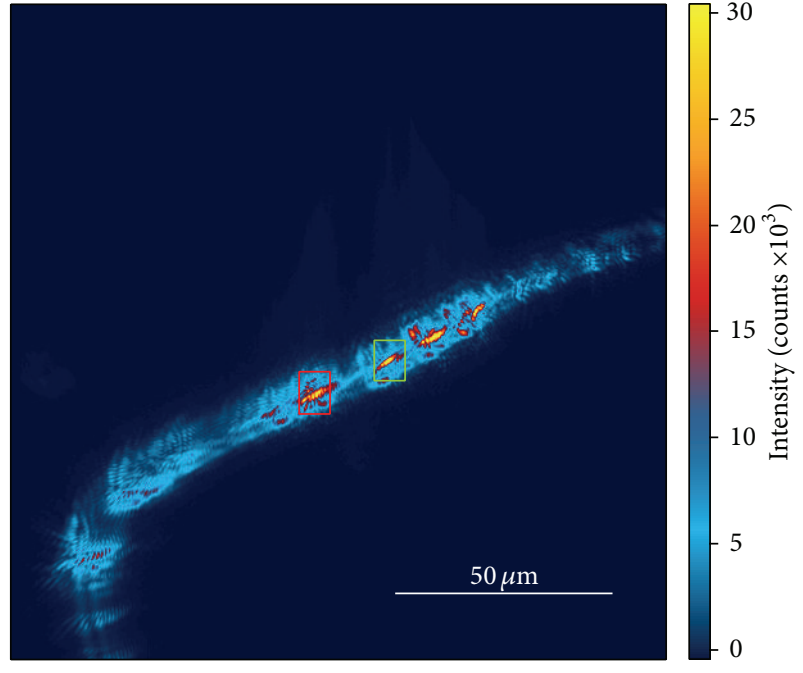

(a)

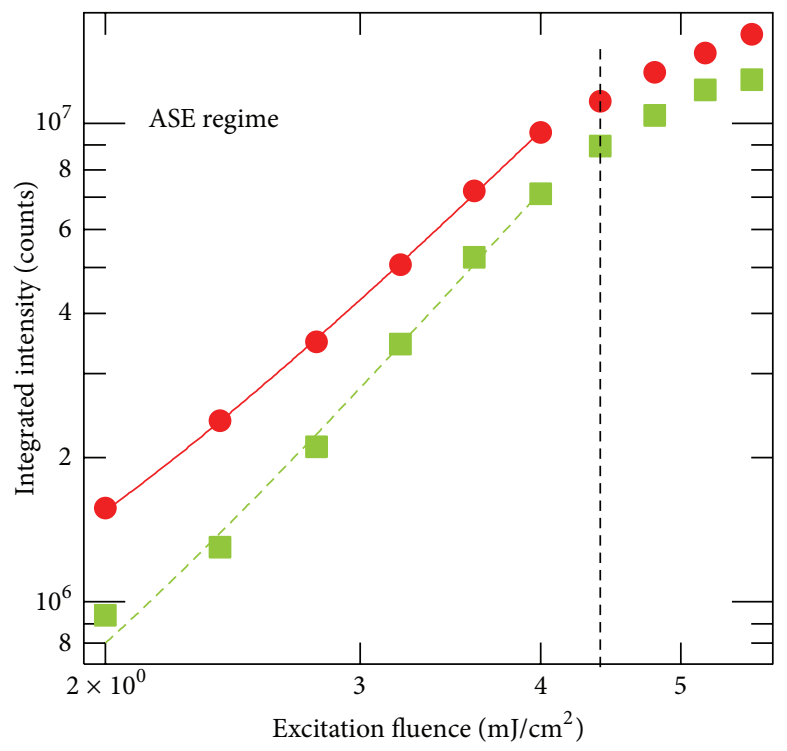

(b)

Figure 2: (a) A time-integrated open gate image displaying the luminescence along the wire in a false-colour scale. (b) The integrated intensity at two different locations along the wire as a function of excitation fluence on a log-log plot.

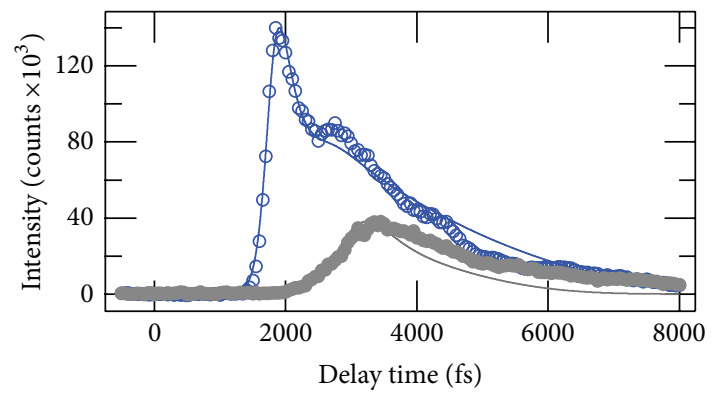

O $4.4 \mathrm{~mJ} / \mathrm{cm}^{2}$ - $2.2 \mathrm{~mJ} / \mathrm{cm}^{2}$

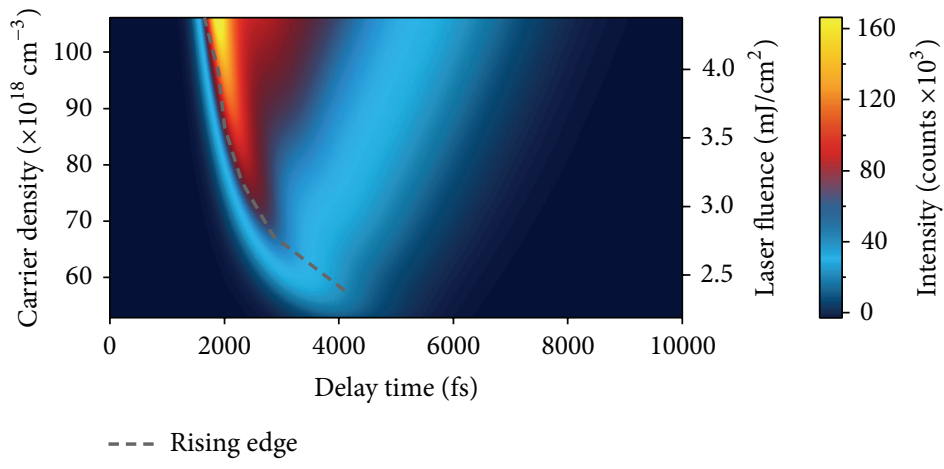

(b)

FIGURE 3: (a) Measured luminescence transients from the nanowire using the Kerr-gated microscope for two different excitation fluences (markers) and the fits from the ASE model (solid lines). (b) The rising edge of the emission with excitation fluence (dashed grey line) on top of the false-colour image of the calculated emission using the ASE model.

where $N_{1}$ and $N_{2}$ are the number of carriers in the initially excited and quasi-relaxed state, respectively, $\Phi(t)$ is the photon field, $V_{p}(t)$ is the Gaussian pump pulse that has an amplitude $A, B$ is the stimulated emission rate, and $\gamma_{r}$ is the relaxation rate from $N_{1}$ to $N_{2}$, while $\gamma_{s}, \gamma_{g}$, and $\gamma_{a}$ are, respectively, the Shockley-Read-Hall (SRH), nongeminate, and Auger rate. The SRH rate provides a measure of the impurity mediated nonradiative lifetime $[13,14]$, while the nongeminate relaxation rate, as shown later on, provides an insight into electron diffusion within the sample.

The results of the model above are shown by the solid lines in Figure 3(a) and the false-colour image in Figure 3(b) (the fitting parameters are as follows: $\gamma_{r}=2.5 \times 10^{11} \mathrm{~s}^{-1}, \gamma_{\Phi}=$ $1.3 \times 10^{13} \mathrm{~s}^{-1}, \gamma_{s}=2.0 \times 10^{-10} \mathrm{~s}^{-1}, \gamma_{g}=3.0 \times 10^{-7} \mathrm{~cm}^{3} \mathrm{~s}^{-1}$, and $\left.\gamma_{a}=3.55 \times 10^{-26} \mathrm{~cm}^{6} \mathrm{~s}^{-1}\right)$. A global fitting routine was used, where the only parameter that changed between the high and low power was the carrier density as given by the increase in the pump laser fluence. From Figure 3(b), it can be seen that the model is excellent in reproducing the shift in the onset of the emission with increasing laser fluence defined as the time when the signal reaches half of the maximum. The model also fits reasonably well the transients, including the appearance of a second peak at high fluences (Figure 3(a)). From these global fits, the extracted Shockley-Read lifetime of 200 ps is comparable to the measured nonradiative recombination lifetimes reported for CdSSe nanobelts [15].

The nongeminate rate from these fits is $3 \times 10^{-7} \mathrm{~cm}^{3} / \mathrm{s}$. Following [16], two different regimes exist depending on 
the mobility of the material. In diffusion limited recombination as found in amorphous semiconductors, $v_{T} \tau_{S} \ll r_{c}$, where $v_{T}$ is the thermal velocity, $\tau_{S}$ is the scattering time, and $r_{c}$ is the Coulomb radius of an oppositely charged carrier. For this regime, the system can be modelled within Langevin recombination [17] where the nongeminate relaxation rate would provide a measure of the Langevin mobility, $\mu_{L}=$ $\mu_{e}+\mu_{h}$ via $\gamma_{g}=e \mu_{L} / \epsilon$ [18], where $\mu_{e}\left(\mu_{h}\right)$ is the electron (hole) mobility, $\epsilon$ is the dielectric constant, and $e$ is the electron charge. For high mobility materials, carrier recombination is not diffusion limited and $v_{T} \tau_{S}>\left(N_{r}\right)^{-1 / 3}$, where $N_{r}$ is the density of carriers. The nongeminate relaxation rate is now given by $\gamma_{g}=v_{T} \sigma$ and $\sigma$ is the capture cross section. Assuming that the diffusion limited regime is present in our undoped ternary alloy nanowires, we calculate a Langevin mobility of $0.2 \mathrm{~cm}^{2} / \mathrm{Vs}$. This value is close to the electron mobility recorded from field-effect-transistor (FET) measurements on pure CdS nanowires $\left(1.7 \mathrm{~cm}^{2} / \mathrm{Vs}\right)$ [19] but several orders of magnitude lower than recent time-resolved terahertz measurements $\left(81.6 \mathrm{~cm}^{2} / \mathrm{Vs}\right)$ on CdSSe nanobelts [15]. Although we do not expect large mobilities in these nanowires, it is likely that at these high carrier densities the carriers recombine before they undergo significant phonon, impurity, or defect scattering, and we do not satisfy the criteria of being in the diffusion limited regime. It can be confirmed by a rough approximation, using a scattering time of $100 \mathrm{fs}$ [20], that at these high carrier densities $v_{T} \tau_{S}$ is approximately equal to $\left(N_{r}\right)^{-1 / 3}$ only when the carrier temperature is on the order of $10 \mathrm{~K}$. Therefore, on these ultrafast timescales and high carrier densities achievable with the Kerr-gated microscope, we can enter the nondiffusion controlled regime. This regime is inaccessible with other techniques that operate at lower densities and longer timescales for low mobility material systems like CdSSe nanowires. We would like to note that many of the nanowires drop-cast on the TEM grids showed similar features as those observed in Figures 2 and 3 . The transients could be reproduced with the above ASE model using a comparable nongeminate rate as that measured for this nanowire.

\section{Conclusions}

Through the study of early luminescence dynamics on ultrashort timescales, we are able to probe carrier dynamics in a single CdSSe nanowire. From fitting the observed timedynamics of the emission, we are able to extract nonradiative recombination rate and the nongeminate recombination constant. From the measurement of the latter, we conclude that on the short timescales available through the use of our Kerr-gated microscope, carrier recombination is not diffusion controlled at high densities even in low mobility materials.

\section{Conflict of Interests}

The authors declare that there is no conflict of interests regarding the publication of this paper.

\section{References}

[1] D. Caselli, Z. Liu, D. Shelhammer, and C.-Z. Ning, "Composition-graded nanowire solar cells fabricated in a single process for spectrum-splitting photovoltaic systems," Nano Letters, vol. 14, no. 10, pp. 5772-5779, 2014.

[2] Y. K. Liu, J. A. Zapien, Y. Y. Shan, H. Tang, C. S. Lee, and S. T. Lee, "Wavelength-tunable lasing in single-crystal $\mathrm{CdS}_{1-X} \mathrm{Se}_{X}$ nanoribbons," Nanotechnology, vol. 18, no. 36, Article ID 365606, 2007.

[3] A. Pan, W. Zhou, E. S. P. Leong et al., "Continuous alloycomposition spatial grading and superbroad wavelengthtunable nanowire lasers on a single chip," Nano Letters, vol. 9, no. 2, pp. 784-788, 2009.

[4] R. K. Ahrenkiel, B. M. Keyes, and D. J. Dunlavy, "Intensitydependent minority-carrier lifetime in III-V semiconductors due to saturation of recombination centers," Journal of Applied Physics, vol. 70, no. 1, pp. 225-231, 1991.

[5] H. W. Liu, J. P. Lu, H. M. Fan, C. H. Sow, S. H. Tang, and X. H. Zhang, "Temperature and composition dependence of photoluminescence dynamics in $\mathrm{CdS}_{x} \mathrm{Se}_{1-x}(0 \leq x \leq 1)$ nanobelts," Journal of Applied Physics, vol. 111, no. 7, Article ID 073112, 2012.

[6] L. Gundlach and P. Piotrowiak, "Femtosecond Kerr-gated widefield fluorescence microscopy," Optics Letters, vol. 33, no. 9, pp. 992-994, 2008.

[7] A. Pan, H. Yang, R. Yu, and B. Zou, "Fabrication and photoluminescence of high-quality ternary CdSSe nanowires and nanoribbons," Nanotechnology, vol. 17, no. 4, pp. 1083-1086, 2006.

[8] J. C. Blake, P. S. Eldridge, and L. Gundlach, "Spatial variation in carrier dynamics along a single CdSSe nanowire," Chemical Physics, vol. 442, pp. 128-131, 2014.

[9] A. Pan, H. Yang, R. Liu, R. Yu, B. Zou, and Z. Wang, "Colortunable photoluminescence of alloyed CdSxSel-x nanobelts," Journal of the American Chemical Society, vol. 127, no. 45, pp. 15692-15693, 2005.

[10] F. Vietmeyer, P. A. Frantsuzov, B. Janko, and M. Kuno, "Carrier recombination dynamics in individual CdSe nanowires," Physical Review B: Condensed Matter and Materials Physics, vol. 83, no. 11, Article ID 115319, 2011.

[11] R. S. Mane and C. D. Lokhande, "Studies on chemically deposited cadmium sulphoselenide (CdSSe) films," Thin Solid Films, vol. 304, no. 1-2, pp. 56-60, 1997.

[12] L. Gundlach and P. Piotrowiak, "Ultrafast spatially resolved carrier dynamics in single cdsse nanobelts," Journal of Physical Chemistry C, vol. 113, no. 28, pp. 12162-12166, 2009.

[13] W. Shockley and W. T. Read, "Statistics of the recombinations of holes and electrons," Physical Review, vol. 87, no. 5, pp. 835-842, 1952.

[14] R. N. Hall, "Electron-hole recombination in germanium," Physical Review, vol. 87, no. 2, p. 387, 1952.

[15] J. Lu, H. Liu, S. X. Lim, S. H. Tang, C. H. Sow, and X. Zhang, "Transient photoconductivity of ternary CDSSE nanobelts as measured by time-resolved terahertz spectroscopy," The Journal of Physical Chemistry C, vol. 117, no. 23, pp. 12379-12384, 2013.

[16] J. Mort, "Geminate and non-geminate recombination in amorphous semiconductors," Le Journal de Physique Colloques, vol. 42, no. C4, pp. C4-433-C4-441, 1981.

[17] P. Langevin, "L'ionization des gaz," Annales de Chimie et de Physique, vol. 28, no. 287, p. 443, 1903. 
[18] A. Rose, Concepts in Photoconductivity and Allied Problems, Interscience Publishers, New York, NY, USA, 1963.

[19] J. S. Jie, W. J. Zhang, Y. Jiang, and S. T. Lee, “Transport properties of single-crystal CdS nanoribbons," Applied Physics Letters, vol. 89, no. 22, Article ID 223117, 2006.

[20] C. Chen, M. Dutta, and M. A. Stroscio, "Electron scattering via interactions with optical phonons in wurtzite crystals," Physical Review B: Condensed Matter and Materials Physics, vol. 70, no. 7, Article ID 075316, 2004. 

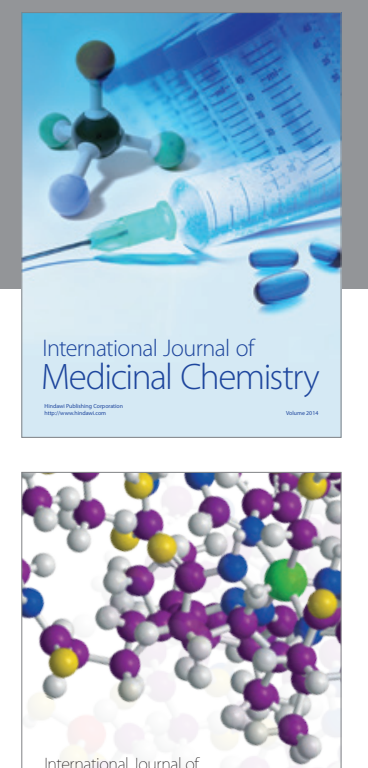

\section{Carbohydrate} Chemistry

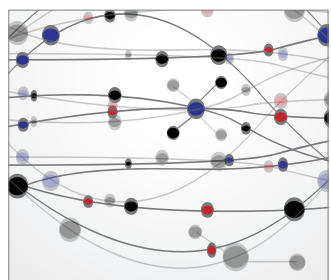

The Scientific World Journal
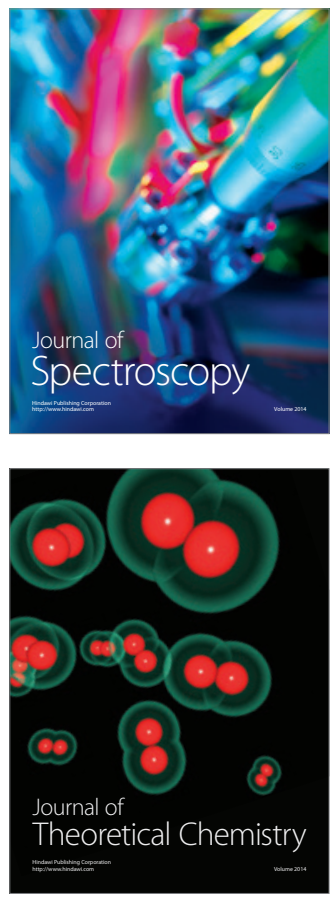
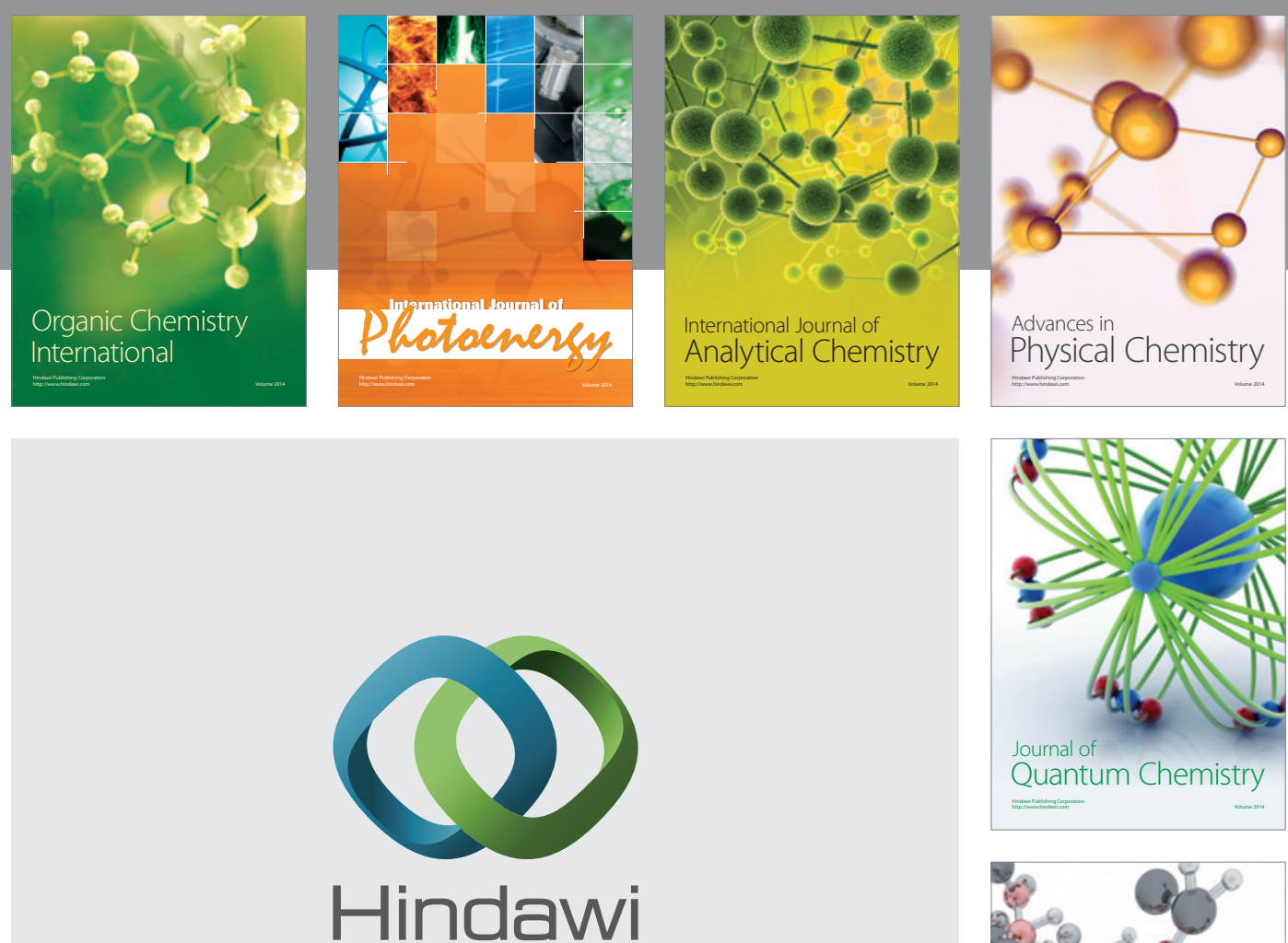

Submit your manuscripts at

http://www.hindawi.com

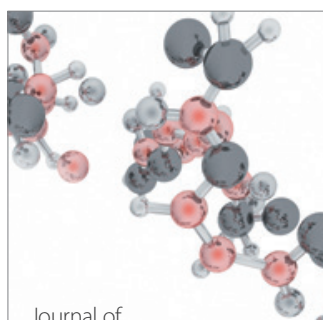

Analytical Methods

in Chemistry

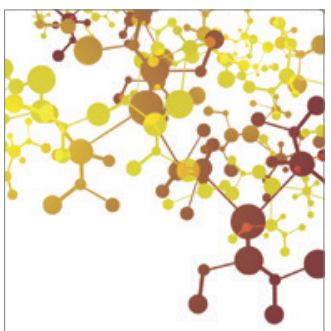

Journal of

Applied Chemistry

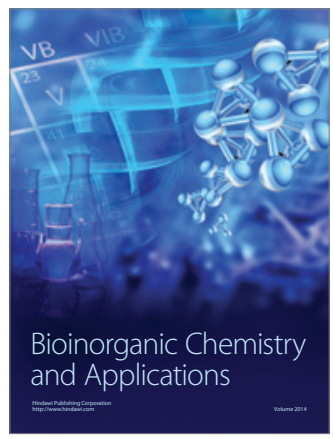

Inorganic Chemistry
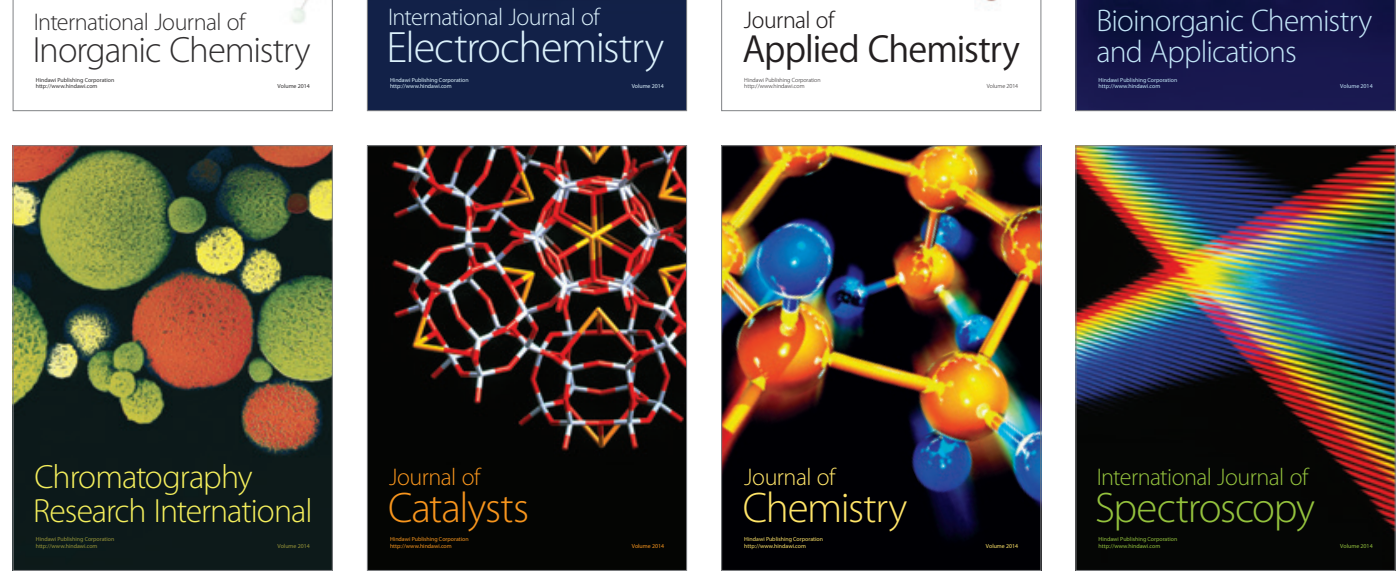